\title{
AVALIAÇÃO DOS EFEITOS DA APLICAÇÃO DE BASALTO MOÍDO NA FERTILIDADE DO SOLO E NUTRIÇÃ̃O DE Eucalyptus benthamii
}

\author{
Alinne da Silva ${ }^{1}$, Jaime Antonio de Almeida ${ }^{2}$, Catiline Schmitt ${ }^{3}$, Cileide Maria Medeiros Coelho ${ }^{4}$ \\ ${ }^{1}$ Enga . Agrônoma, Doutoranda em Ciência, USP, Piracicaba, SP, Brasil - alinnesilva2000@yahoo.com \\ ${ }^{2}$ Eng. Agrônomo, Dr., Depto. de Solos, UDESC, Lages, SC, Brasil - a2jaa@cav.udesc.br \\ ${ }^{3} \mathrm{Eng}^{\mathrm{a}}$. Agrônoma, Doutoranda em Ciência do Solo, UFRGS, Porto Alegre, RS, Brasil - s_katiline@yahoo.com.br \\ ${ }^{4}$ Eng $^{\mathrm{a}}$. Agrônoma, Dr ${ }^{\mathrm{a}}$., Depto. de Agronomia, UDESC, Lages, SC, Brasil - a2cmm @ cav.udesc.br \\ Recebido para publicação: 06/06/2011 - Aceito para publicação: 08/11/2011
}

\begin{abstract}
Resumo
Considerando a necessidade de avaliar a eficácia do pó de basalto como fonte de nutrientes e o efeito da adição de estercos animais sobre a dissolução desses materiais, foi instalado um experimento em casa de vegetação. Os tratamentos aplicados foram: controle; adubação convencional; 10,0, 20,0, 50,0 e 100,0 t.ha ${ }^{-1}$ de pó de basalto; e as mesmas doses de pó de basalto associado ao esterco bovino. Os tratamentos foram testados em mudas de clone de Eucalyptus benthamii. Todos os tratamentos foram comparados ao controle pelo teste de Duncan. A aplicação de pó de basalto promoveu aumento no valor do $\mathrm{pH}$ do solo para todas as doses. Os teores de $\mathrm{Mg}$ e Si trocável no solo foram maiores nos tratamentos com pó de basalto associado ao esterco bovino, o qual demonstrou ser um material que interfere na dissolução dos minerais e liberação desses elementos. $\mathrm{O}$ pó de basalto, em associação ou não com esterco bovino, conferiu teores de $\mathrm{K}$ nas folhas maiores que o tratamento controle. Concluise que doses a partir de 20 t.ha $^{-1}$ de pó de basalto associadas ao esterco bovino apresentaram efeito no $\mathrm{pH}$ do solo e podem ser utilizadas como fonte de $\mathrm{Mg}$, Si e K.

Palavras-chave: Rochagem; minerais; fontes de nutrientes.
\end{abstract}

\begin{abstract}
Evaluation of application of basalt powder effects in soil fertility and Eucalyptus benthamii nutrition. In order to evaluate powdered basalt efficiency as a source of nutrients as well as effects of cattle manure on dissolution of these materials, it was improved an experiment in a greenhouse. The improved treatments were: control; conventional fertilization; 10.0, 20.0, 50.0 and $100.0 \mathrm{t} \mathrm{ha}^{-1}$ of basalt powder, and the same basalt powder doses associated with cattle manure. Such treatments had been tested on Eucalyptus benthamii seedlings. All treatments were compared to control by Duncan test. Basalt powder application promoted soil $\mathrm{pH}$ increasing in every doses. The contents of exchangeable $\mathrm{Mg}$ and $\mathrm{Si}$ were higher in treatments with powdered basalt associated with cattle manure, which proved to be a material that interferes in minerals dissolution as well as in release of such elements. The basalt powder, in association or not with cattle manure, imporved $\mathrm{K}$ contents in the leaves higher than the control ones. We can conclude that doses from $20 \mathrm{t} \mathrm{ha}^{-1}$ of basalt powder associated with cattle manure have effect on soil $\mathrm{pH}$ and can be use as a source of $\mathrm{Mg}$, $\mathrm{Si}$ and $\mathrm{K}$. Keywords: Stonemeal; minerals; source of nutrients.
\end{abstract}

\section{INTRODUÇÃO}

As áreas de florestas plantadas com eucalipto no Brasil ocuparam 4.515.730 ha em 2009 (ASSOCIAÇÃO BRASILEIRA DE PRODUTORES DE FLORESTAS PLANTADAS (ABRAF), 2010), sendo parte dessa área situada em locais com risco de ocorrência de geadas, como em algumas regiões dos estados do Sul do país, onde as opções de plantio são restritas a poucas espécies que apresentam resistência ao frio, como Eucalyptus benthamii Maiden et Cambage (HIGA, 1999; GRAÇA et al., 1999).

E. benthamii é uma planta nativa da Austrália, onde, originalmente, ocorre em solos férteis. Desse modo, para a espécie manifestar seu potencial produtivo, ela necessita que os elementos essenciais estejam disponíveis no solo para serem absorvidos. Assim, considerando a baixa fertilidade da maioria 
dos solos brasileiros, as práticas de calagem e adubação são indispensáveis para fornecer nutrientes que não estão em concentrações satisfatórias para atender a demanda das plantas nas áreas de reflorestamentos. Contudo, o alto custo dos corretivos e adubos convencionais fortalece a demanda por possíveis fontes alternativas de nutrientes em áreas destinadas à silvicultura.

Uma das alternativas viáveis aos fertilizantes convencionais pode ser o uso de rochas moídas e aplicadas ao solo na forma de pó, prática denominada rochagem. Leonardos et al. (1976) indicam para a prática da rochagem, entre outros, o basalto, que apresenta em sua composição macronutrientes, micronutrientes e elementos benéficos que podem contribuir com o efeito residual por um longo período (HARLEY; GILKES, 2000).

Para se tornar fonte de nutrientes, os minerais do pó de rocha devem sofrer processos de intemperismo (STRAATEN, 2002). Esses processos são complexos e dependem principalmente da composição química e mineralógica da rocha, granulometria, tempo de reação e fatores do solo, como pH e atividade biológica. Portanto, a associação dos pós de rocha com materiais que apresentem grande atividade biológica, tais como os estercos animais, pode influenciar no processo de alteração dos minerais, porém pouco se sabe sobre o efeito desses materiais sobre a dissolução das rochas moídas.

Alguns autores questionam a eficiência do pó de rocha como fonte de nutrientes devido à baixa solubilidade (BOLLAND; BAKER, 2000), porém uma série de estudos comprova os efeitos positivos do uso de rochas moídas como fonte de nutrientes para o solo. Knapik e Angelo (2007) avaliaram o desenvolvimento de Prunus sellowii Koehne e observaram que o melhor crescimento das mudas foi nos tratamentos com NPK. As mudas produzidas com pó de basalto não apresentaram crescimento similar àquelas que receberam adubação convencional, no entanto, nesses tratamentos foi observado maior teor de cálcio $(\mathrm{Ca})$, magnésio $(\mathrm{Mg})$, boro $(\mathrm{B})$, cobre $(\mathrm{Cu})$ e ferro $(\mathrm{Fe})$ nas folhas. Theodoro e Leonardos $(2006)$ avaliaram o potencial de rocha vulcânica em um solo arenoso e verificaram aumento do $\mathrm{pH}$ e dos teores de $\mathrm{Ca}, \mathrm{Mg}$, fósforo $(\mathrm{P})$ e potássio $(\mathrm{K})$ após o primeiro ano, permanecendo com teores maiores aos das parcelas que não receberam o pó mesmo após cinco anos.

O basalto moído pode representar uma alternativa aos fertilizantes convencionais como fonte de nutrientes e a sua associação com materiais que apresentam grande atividade biológica, tais como os estercos animais, pode influenciar no processo de alteração dos minerais e na consequente liberação dos elementos para o solo. Visando testar essa hipótese, foi instalado um experimento para comparar quatro doses de pó de basalto, associadas ou não ao esterco bovino, a um tratamento controle, que não recebeu pó de basalto. O objetivo foi avaliar a eficiência da rocha moída sobre o $\mathrm{pH}$ e os valores de $\mathrm{K}, \mathrm{Ca}, \mathrm{Mg}$ e silício ( $\mathrm{Si}$ ) no solo, o efeito da adição de esterco bovino sobre a dissolução dos minerais presentes nessa rocha e os efeitos na nutrição em mudas de E. Benthamii.

\section{MATERIAL E MÉTODOS}

O Eucalyptus benthamii é de origem australiana, pertence à família Myrtaceae e é encontrado em áreas restritas no oeste da cidade de Sydney, em planícies ao longo do Rio Nepean e no vale do Blue Mountains National Park (HIGA; PEREIRA, 2003). E. benthamii foi introduzido no Brasil pela Empresa Brasileira de Pesquisa Agropecuária (EMBRAPA) Florestas de Colombo, no estado do Paraná, e os estudos experimentais têm revelado que essa espécie apresenta resistência a geadas, rápido crescimento e alta homogeneidade do talhão (GRAÇA et al., 1999).

O experimento foi instalado em fevereiro de 2006, em casa de vegetação no município de Lages, SC (27 $48^{\circ} 57^{\prime \prime}$ S e $50^{\circ} 19^{\prime} 33^{\prime}$ ' O). O solo do experimento pertence à classe Nitossolo Bruno Distrófico típico (EMBRAPA, 2006), desenvolvido de basalto e com 65\% de argila no horizonte A, e apresentava as seguintes características: $\mathrm{pH}$ em água $=4,7, \mathrm{pH}$ SMP $=5,0, \mathrm{P}$ Mehlich-1 $=1,0 \mathrm{mg} \cdot \mathrm{dm}^{-3}, \mathrm{~K}=161 \mathrm{mg} \cdot \mathrm{dm}^{-3}$, $\mathrm{Al}^{3+}=2,7 \mathrm{cmol}_{\mathrm{c}} \cdot \mathrm{dm}^{-3}, \mathrm{Ca}=1,6 \mathrm{cmol}_{\mathrm{c}} \cdot \mathrm{dm}^{-3} \mathrm{e} \mathrm{Mg}=1,0 \mathrm{cmol}_{\mathrm{c}} \cdot \mathrm{dm}^{-3}$.

O solo foi coletado no município de Urupema, SC (27 $57^{\prime} 20^{\prime \prime}$ S e $49^{\circ} 52^{\prime} 34$ '” O), na camada entre 0 e $20 \mathrm{~cm}$, e corrigido com calcário para elevação do pH até 5,5. Com exceção do tratamento com adubação convencional, nos demais tratamentos, inclusive no controle, foram adicionadas $1 \mathrm{t}^{-\mathrm{ha}^{-1}}$ de pó de granito feldspático como fonte de $\mathrm{K}$, o qual apresenta uma média de $54,6 \mathrm{~g} \cdot \mathrm{kg}^{-1} \mathrm{de} \mathrm{K}_{2} \mathrm{O}$ e $300 \mathrm{~kg} \cdot \mathrm{ha}^{-1}$ de fosfato natural de Araxá como fonte de $\mathrm{P}$, que apresenta uma média de $300 \mathrm{~g} \cdot \mathrm{kg}^{-1}$ de $\mathrm{P}_{2} \mathrm{O}_{5}$. Após a adição desses materiais, o solo passou a ter as seguintes características: $\mathrm{pH}$ em água $=5,5 ; \mathrm{Al}=0,86 \mathrm{cmol}_{\mathrm{c}} \cdot \mathrm{dm}^{-3} ; \mathrm{K}=$ 164,17 mg.dm ${ }^{-3} ;$ P Mehlich-1 = 45,58 mg.dm ${ }^{-3} ; \mathrm{Ca}=8,34 \mathrm{cmol}_{\mathrm{c}} \cdot \mathrm{dm}^{-3} ; \mathrm{e} \mathrm{Mg}=1,45 \mathrm{cmol}_{\mathrm{c}} \cdot \mathrm{dm}^{-3}$. 
O basalto utilizado como fonte de macro e micronutrientes foi proveniente da pedreira Manjolinho, situada em Ponte Alta (SC). Em laudo técnico de análise petrográfica fornecido pela pedreira, constatou-se que o basalto é do tipo meláfiro, com relevante estado de argilização e formação de óxidos de ferro por toda a sua porção central. Apresenta estrutura maciça, contendo plagioclásios do tipo labradorita $\left(\mathrm{Na}_{0,5-0,3}, \mathrm{Ca}_{0,5}\right.$ $\left.{ }_{0,7}\right) \mathrm{Al}\left(\mathrm{Al}_{0,5-0,7,7}, \mathrm{Si}_{0,5-0,3}\right) \mathrm{Si}_{2} \mathrm{O}_{8}$, clinopiroxênio do tipo augita $(\mathrm{Ca}, \mathrm{Mg}, \mathrm{Al})_{2}(\mathrm{Si}, \mathrm{Al})_{2} \mathrm{O}_{6}$, opacos de pirita $\left(\mathrm{FeS}_{2}\right)$ e ilmenita $\left(\mathrm{FeTiO}_{2}\right)$ e óxidos de ferro. $\mathrm{O}$ basalto foi recolhido na forma de brita, submetido à moagem em moinho de martelo e posteriormente passado em peneira com malha de $2 \mathrm{~mm}$. Os teores de elementos da referida rocha foram quantificados após digestão por fusão total em laboratório com controle de qualidade internacional no Canadá (Actlabs), cujos resultados estão indicados na tabela 1.

Tabela 1. Teores de elementos presentes no basalto utilizado no experimento.

Table 1. Concentrations of elements present in the basalt used in the experiment.

\begin{tabular}{|c|c|c|c|c|c|c|c|c|c|c|}
\hline $\mathrm{SiO}_{2}$ & $\mathbf{A l}_{2} \mathbf{O}_{3}$ & $\mathrm{Fe}_{2} \mathrm{O}_{3}$ & $\mathrm{MnO}$ & MgO & $\mathrm{CaO}$ & $\mathrm{Na}_{2} \mathrm{O}$ & $\mathrm{K}_{2} \mathrm{O}$ & $\mathrm{TiO}_{2}$ & $\mathbf{P}_{2} \mathbf{O}_{5}$ & LOI \\
\hline 50,37 & 13,20 & 13,28 & 0,167 & 4,82 & 8,56 & 2,65 & 1,44 & 3,57 & 0,45 & 1,30 \\
\hline $\mathbf{B a}$ & $\mathbf{S r}$ & $\mathbf{Y}$ & Sc & $\mathbf{Z r}$ & $\mathbf{B e}$ & $\mathbf{V}$ & As & Co & Mo & Massa \\
\hline \multirow[t]{3}{*}{568} & 720 & 32 & 23 & 257 & 2 & 347 & $<0,5$ & 40 & $<5$ & 1,42 \\
\hline & & $\mathbf{C d}$ & $\mathbf{C u}$ & $\mathrm{Ni}$ & $\mathbf{Z n}$ & $\mathbf{S}$ & $\mathbf{A g}$ & $\mathbf{P b}$ & & \\
\hline & & $<0,5$ & 184 & 66 & 100 & 0,004 & $<0,3$ & $<5$ & & \\
\hline
\end{tabular}

Foram utilizadas mudas de clone de Eucalyptus benthamii com cerca de $15 \mathrm{~cm}$ de altura da parte aérea e 3 a $4 \mathrm{~mm}$ de diâmetro de colo, disponibilizadas pela Empresa de Pesquisa Agropecuária e Extensão Rural de Santa Catarina (EPAGRI). As mudas foram plantadas em vasos de polietileno com volume de $8 \mathrm{~L}$, mantidas com $80 \%$ da capacidade máxima de retenção de umidade entre os meses de fevereiro e dezembro de 2006.

O delineamento experimental foi inteiramente casualizado (DIC), com três repetições. Os tratamentos se constituíram das seguintes combinações: controle com pó de granito $\left(1 \mathrm{t}^{-1} \mathrm{~h}^{-1}\right)$ e fosfato natural $\left(300 \mathrm{~kg} \mathrm{ha}^{-1}\right)$ (controle); adubação convencional com NPK (AC); doses de 10,0, 20,0, 50,0 e 100,0 t.ha ${ }^{-1}$ de pó de basalto associadas ao pó de granito $\left(1 \mathrm{t}^{-h^{-1}}\right)$ e ao fosfato natural $\left(300 \mathrm{~kg} \cdot \mathrm{ha}^{-1}\right)(\mathrm{PB})$; e as mesmas doses de pó de basalto associado ao esterco bovino (4 t.ha ${ }^{-1}$ base seca), pó de granito e fosfato natural $(\mathrm{PB}+\mathrm{EB})$, totalizando dez tratamentos. $\mathrm{O}$ tratamento com adubação convencional recebeu adubo solúvel nas quantidades de $105 \mathrm{~kg} \cdot \mathrm{ha}^{-1}$ de $\mathrm{P}_{2} \mathrm{O}_{5}, 30 \mathrm{~kg} \cdot \mathrm{ha}^{-1}$ de $\mathrm{K}_{2} \mathrm{O}$ e $30 \mathrm{~kg} \cdot \mathrm{ha}^{-1}$ de ureia, de acordo com as recomendações da Comissão de Química e Fertilidade do Solo (CQFSRS/SC) (2004).

A fertilidade do solo foi avaliada pelas seguintes determinações: $\mathrm{pH}$ em água, $\mathrm{K}, \mathrm{Ca}$ e $\mathrm{Mg}$, de acordo com metodologias descritas por Tedesco et al. (1995). A determinação do Si trocável do solo foi realizada segundo Elliott e Snyder (1991).

$\mathrm{O}$ estado nutricional do eucalipto foi determinado pela coleta de 10 folhas do terço superior de cada planta em dezembro de 2006, conforme proposto por Silveira et al. (2000). Os autores sugerem que a amostragem foliar para a avaliação do estado nutricional do Eucaliptus sp. deve ser realizada entre os 12 e 18 meses após o plantio, pela coleta das primeiras folhas completamente desenvolvidas dos ramos situados no terço superior da planta.

As folhas foram acondicionadas em sacos de papel e mantidas em estufa com circulação forçada de ar a $60{ }^{\circ} \mathrm{C}$ por 72 horas, até atingir peso constante. Após esse período, as folhas foram moídas em moinho de faca tipo Wiley e posteriormente passadas em peneira de $1 \mathrm{~mm}$. Foram determinadas as concentrações de $\mathrm{K}, \mathrm{Ca}, \mathrm{Mg}, \mathrm{Zn}, \mathrm{Cu}, \mathrm{Mn}$ e Fe de acordo com metodologias descritas por Tedesco et al. (1995). A determinação do Si na planta foi realizada segundo procedimento descrito por Elliott e Snyder (1991).

Os dados foram submetidos à análise de variância pelo teste $\mathrm{F}(\mathrm{p} \leq 0,05)$ e, quando significativo, todos os tratamentos foram comparados com o tratamento-testemunha pelo teste Duncan $(\mathrm{p} \leq 0,05)$ utilizando-se o programa estatístico SAS (2007). 


\section{RESULTADOS E DISCUSSÃO}

\section{Efeito do pó de basalto sobre propriedades químicas do solo}

Foram verificadas respostas positivas pela aplicação do pó de basalto para o $\mathrm{pH}$, teores de $\mathrm{Mg}$ e Si trocáveis do solo (Tabela 2).

$\mathrm{O}$ pH do solo aumentou em função da aplicação de pó de basalto. Os tratamentos que receberam as doses 10, 20, 50 e 100 t.ha $^{-1}$ de PB, com ou sem associação ao esterco bovino, apresentaram diferença significativa quando comparados com o controle (Tabela 2). $\mathrm{O} \mathrm{pH}$ do solo, que foi corrigido para 5,5 antes da instalação do experimento, passou a apresentar valor de 6,1 na média dos quatro tratamentos pela aplicação do PB e 6,3 na média dos quatro tratamentos com PB+EB (Tabela 2). Esse aumento nos valores de $\mathrm{pH}$ é uma característica importante do pó de rocha, pois a elevação do $\mathrm{pH}$ do solo, além de estimular a atividade microbiológica (STEVENSON; COLE, 1999), pode diminuir as perdas de K por lixiviação, devido à criação de cargas negativas (QUAGGIO, 2000).

Essa elevação no valor do $\mathrm{pH}$ pode ser atribuída às quantidades de $\mathrm{CaO}$ e de $\mathrm{MgO}$ presentes no basalto, 8,56 e 4,82\%, respectivamente (Tabela 1). Knapik e Angelo (2007) estudaram o crescimento de mudas de Prunus sellowii submetidas à adubação convencional e, como alternativa, pó de basalto, e encontraram o maior valor de $\mathrm{pH}$ no tratamento com pó de basalto. Segundo os autores, a elevação do $\mathrm{pH}$ deve-se às maiores quantidades de $\mathrm{Ca}$ e $\mathrm{Mg}$ adicionadas ao substrato pelo pó de rocha.

É característica do pó de rochas básicas ou alcalinas promoverem aumento no pH do solo, como já demonstrado por Gillman (1980) e Gillman et al. (2001) pela aplicação de pó de rochas basálticas em solo de baixa fertilidade química de floresta. Gillman (1980) incubou por 12 meses pó de basalto no solo e observou aumento significativo no $\mathrm{pH}$ e na capacidade de troca de cátions (CTC), constatando que o efeito foi mais pronunciado com a diminuição da granulometria e aumento do tempo de contato entre o material e o solo. Gillman et al. (2001) observaram o comportamento de sete tipos de solos com a incubação de doses de pó de basalto $\left(0,1,5,25\right.$ e 50 t.ha $\left.^{-1}\right)$, verificando aumento no $\mathrm{pH}$ e nos teores de cátions básicos.

Apesar da adição de $\mathrm{CaO}$ e $\mathrm{MgO}$ pelo pó de basalto, não houve aumento concomitante nos teores de $\mathrm{Ca}$ nos tratamentos em que foram aplicados $\mathrm{PB}$, conforme seria esperado, o que pode ser devido à liberação de hidroxilas durante a dissolução dos minerais por hidrólise, sem que tenha havido aumento expressivo na quantidade total desse cátion básico trocável. Porém, nos tratamentos PB+EB foi observado que os teores de $\mathrm{Mg}$ foram maiores que os do tratamento-controle (Tabela 2).

Nesses tratamentos pode ter ocorrido decomposição de minerais menos resistentes à alteração química, pois se trata de uma rocha ferromagnesiana rica em minerais facilmente alteráveis (RESENDE et al., 2002), aliados à presença do esterco bovino, que pode ter sido fator preponderante na aceleração do processo de decomposição desses minerais, contribuindo para o aumento da atividade biológica do solo, característica marcante também na rizosfera das plantas (MARSCHNER, 1991). Theodoro e Leonardos (2006) observaram que o uso adicional de composto orgânico foi vantajoso, mostrando que a combinação da fertilização orgânica e da rocha pode cumprir, se não todas, a maioria das exigências de macro e micronutrientes.

Os teores de K no solo não aumentaram pela aplicação de nenhuma das doses de PB e PB+EB, bem como pela aplicação de AC. Mesmo esta sendo uma fonte prontamente solúvel, o $\mathrm{KCl}$ não apresentou valores de $\mathrm{K}$ maiores que o controle. Isso se deve ao pó de granito que foi aplicado como fonte de $\mathrm{K}$ inclusive no tratamento-controle e pode ter uniformizado os teores do elemento em todos os tratamentos. Esses resultados indicam que o pó de granito foi capaz de liberar K para o solo, apesar da solubilidade lenta, pois os minerais que fazem parte da composição do granito são mais resistentes ao intemperismo quando comparados aos do basalto (FRAGSTEIN et al., 1988).

Essa disponibilização lenta e gradual de K é vantajosa, uma vez que pode atender a demanda das plantas por um longo período e reduzir eventuais perdas por lixiviação, evitando dessa forma a contaminação dos recursos hídricos, pois, apesar de o K não ser poluente das águas subsuperficiais, a alta concentração do elemento favorece a formação de pares iônicos, que, com carga neutra, são facilmente lixiviados (QAFOKU et al., 2000). Dessa forma, o par iônico formado entre o $\mathrm{K}^{+}$e o nitrato $\left(\mathrm{NO}_{3}^{-}\right)$é preocupante do ponto de vista ambiental, pois o $\mathrm{NO}_{3}{ }^{-}$é poluente das águas.

Wilpert e Lukes (2003) descrevem efeitos positivos do pó de rocha sobre incremento nos teores de K em solo sob floresta na Alemanha pela aplicação de 6 t.ha ${ }^{-1}$ de pó de rocha vulcânica. Escosteguy e 
Klant (1998) observaram aumentos na concentração de K e Ca em um Latossolo Vermelho e em um Podzólico Vermelho-Amarelo pela aplicação de doses até 100 t.ha $^{-1}$ de pó de basalto em um período que compreendeu 300 dias. Verificaram ainda que as concentrações de $\mathrm{K}$ foram maiores com o aumento do período de incubação.

Tabela 2. Valores de pH em água, teores de $\mathrm{Ca}, \mathrm{Mg}, \mathrm{K}$ e Si trocáveis do solo em função dos tratamentos empregados.

Table 2. Soil $\mathrm{pH}$, exchangeable $\mathrm{Ca}, \mathrm{Mg}$, Si and $\mathrm{K}$ according to the treatments.

\begin{tabular}{lccccc}
\hline \multirow{2}{*}{ Tratamentos } & $\mathbf{p H}$ & $\mathbf{C a}$ & $\mathbf{M g}$ & $\mathbf{K}$ & $\mathbf{S i}$ \\
\cline { 2 - 6 } & \multicolumn{3}{c}{$\left(\mathbf{c m o l}_{\left.\mathbf{c} \cdot \mathbf{d m}^{-3}\right)}\right.$} & \multicolumn{2}{c}{$\left(\mathbf{m g . d m}^{-\mathbf{3}}\right)$} \\
\hline Controle & 5,5 & 6,12 & 1,36 & 133,67 & 9,28 \\
AC & $5,9^{*}$ & 5,16 & 1,42 & 165,50 & 7,23 \\
10 t.ha $^{-1} \mathrm{~PB}$ & $6,2^{*}$ & 5,60 & 1,52 & 141,50 & 8,56 \\
20 t.ha $^{-1} \mathrm{~PB}$ & $6,0^{*}$ & 6,04 & 1,42 & 146,50 & 9,19 \\
50 t.ha $^{-1} \mathrm{~PB}$ & $6,1^{*}$ & 2,80 & 1,46 & 182,50 & 9,37 \\
100 t.ha $^{-1} \mathrm{~PB}$ & $6,0^{*}$ & 4,12 & 1,42 & 186,50 & 9,78 \\
10 t.ha $^{-1} \mathrm{~PB}+\mathrm{EB}$ & $6,4^{*}$ & 4,36 & 1,70 & 154,66 & $15,19^{*}$ \\
20 t.ha $^{-1} \mathrm{~PB}+\mathrm{EB}$ & $6,0^{*}$ & 5,48 & $1,78^{*}$ & 166,00 & $14,61^{*}$ \\
50 t.ha \\
-1 PB + EB & $6,3^{*}$ & 4,04 & $1,72^{*}$ & 138,33 & $12,74^{*}$ \\
100 t.ha $^{-1} \mathrm{~PB}+\mathrm{EB}$ & $6,4^{*}$ & 5,04 & $1,72^{*}$ & 185,50 & $13,36^{*}$ \\
C.V.\% & 3,02 & 44,75 & 9,21 & 13,52 & 11,62 \\
\hline
\end{tabular}

Médias seguidas de * diferem do controle pelo teste de Dunnet $(\mathrm{p} \leq 0,05)$.

Do mesmo modo que ocorreu com o $\mathrm{Mg}$, o esterco associado ao pó das rochas também propiciou aumento no teor de Si trocável no solo (Tabela 2). Os tratamentos com PB+EB apresentaram teores de $\mathrm{Si}$ superiores quando comparados ao controle $\left(9,28 \mathrm{mg} \cdot \mathrm{dm}^{-3}\right)$, ao tratamento com AC $\left(7,23 \mathrm{mg}^{\mathrm{dm}} \mathrm{dm}^{-3}\right)$ e aos tratamentos com PB sem EB $\left(9,22 \mathrm{mg} \cdot \mathrm{dm}^{-3}\right)$.

De acordo com esses resultados, conclui-se que o pó de basalto pode ser empregado como fonte de $\mathrm{Mg}$ e Si para o solo, pois apresenta na sua composição vários minerais silicatados, como piroxênios, plagioclásios e minerais ferromagnesianos facilmente alteráveis, como já referido por outros autores (RESENDE et al., 2002), e que a combinação entre o pó da rocha e o esterco demonstrou ter influenciado na velocidade de liberação desses minerais, tornando o processo mais rápido e, consequentemente, a liberação do elemento para o solo.

Do ponto de vista biológico, os estercos de animais apresentam uma grande quantidade de microrganismos e sabe-se que eles produzem várias substâncias (KIEHL, 1985), as quais podem acelerar, pelo intemperismo biológico, a liberação dos elementos que fazem parte da composição mineralógica da rocha.

\section{Efeito do pó de basalto sobre a nutrição do eucalipto}

A aplicação de pó de basalto, associada ou não ao esterco bovino, proporcionou maiores teores de K e Si nas folhas de E. benthamii (Tabela 3), o que confere benefícios do ponto de vista fisiológico para a planta.

O K está envolvido nos mecanismos de defesa das plantas a pragas e doenças, uma vez que altos teores desse elemento nos tecidos favorecem a síntese e o acúmulo de compostos fenólicos (PERRENOUD, 1990), conferindo às plantas redução na incidência e severidade de danos causados por insetos e fungos. Da mesma forma o Si, que, apesar de ser classificado como elemento não essencial, pode conferir inúmeros benefícios à planta, tais como o aumento na eficiência da capacidade fotossintética, pois as folhas ficam mais eretas, com diminuição do autossombreamento, redução no acamamento, maior rigidez estrutural dos tecidos e resistência a insetos e doenças (MARSCHNER, 1995).

Os teores de $\mathrm{K}$ nas folhas do eucalipto dos tratamentos que receberam 10, 50 e 100 t.ha $^{-1}$ de PB e 50 e 100 t.ha $^{-1} \mathrm{~PB}+\mathrm{EB}$ foram superiores em relação ao tratamento-controle (Tabela 4), embora não tenha sido observado o mesmo resultado com as mesmas doses de PB e PB+EB no solo, os quais não foram superiores ao controle, tratamento que também recebeu pó de granito (Tabela 2). 
Como exposto anteriormente, a aplicação de pó de granito pode ter uniformizado os teores do elemento no solo em todos os tratamentos que receberam o material, contudo, os tratamentos em que foi aplicado o PB apresentaram teor de $\mathrm{K}$ nas folhas maior que o tratamento-controle, pois os minerais que fazem parte da composição do basalto são menos resistentes ao intemperismo quando comparados aos do granito, segundo Fragstein et al. (1988), que observaram que as rochas basálticas apresentam taxas de liberação de elementos minerais mais rápidas quando comparadas com o granito. A granulometria também tem grande influência, pois, quanto maior a área superficial exposta ao ataque dos agentes químicos e biológicos do intemperismo, mais rápida é a alteração do material (OSTERROHT, 2003), favorecendo, portanto, o basalto, rocha de textura afanítica, em relação ao granito, rocha de textura fanerítica.

O emprego de pó de rochas em solo de florestas representa alternativa viável do ponto de vista econômico para ser utilizada em substituição ao adubo potássico convencional, uma vez que o pó de basalto, assim como o AC, proporcionou suprimento adequado de $\mathrm{K}$ para as plantas de eucalipto, que apresentaram conteúdo de $\mathrm{K}$ nas folhas, nos tratamentos com pó de rocha, em média de $12,27 \mathrm{~g}^{\mathrm{kg}} \mathrm{kg}^{-1}$ (Tabela 3), dentro da faixa de suficiência para a cultura $\left(9\right.$ a $\left.13 \mathrm{~g} \mathrm{~kg}^{-1}\right)$, segundo Gonçalves (1995). Dessa forma, pode ser considerada alternativa de substituição ao $\mathrm{KCl}$, visto que cerca de $92 \%$ do $\mathrm{K}$ consumido pelo Brasil é importado (ASSOCIAÇÃO NACIONAL PARA A DIFUSÃO DE ADUBOS (ANDA), 2009), gerando um alto custo de produção.

Em relação ao $\mathrm{Si}$, embora os tratamentos com 10, 20, 50 e 100 t.ha $^{-1}$ de $\mathrm{PB}+\mathrm{EB}$ tenham apresentado valores de Si trocável no solo superior ao tratamento-controle, os teores desse elemento nas folhas de

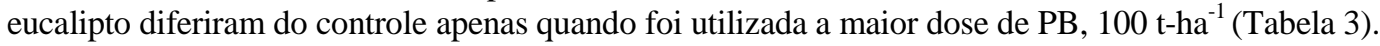

$\mathrm{O}$ conteúdo de $\mathrm{Ca}$ e $\mathrm{Mg}$ nas folhas de eucalipto nos tratamentos com $\mathrm{PB}$, associados ou não ao esterco, não foram maiores do que os obtidos no controle, ainda que os tratamentos com $\mathrm{PB}+\mathrm{EB}$ tenham apresentado os maiores valores de Mg. Ferreira et al. (2009), estudando os mesmos pós de rochas aplicados em um Cambissolo Húmico, também não observaram aumento nos teores desses elementos nas plantas de feijão. Os autores interpretaram a ausência de diferenças entre os tratamentos que receberam o pó de basalto como decorrente da aplicação do calcário para elevar o $\mathrm{pH}$ 5,2, em todos os tratamentos, e ao curto período de aplicação dos mesmos, já que se trata de material de baixa solubilidade.

Tabela 3. Teor de macronutrientes, micronutrientes e silício nas folhas de eucalipto de acordo com os tratamentos.

Table 3. Macronutrients, micronutrients and silicon measures in eucalyptus leaves accordingly to the treatments.

\begin{tabular}{|c|c|c|c|c|c|c|c|c|}
\hline \multirow{2}{*}{ Tratamentos } & $\mathbf{K}$ & $\mathbf{C a}$ & Mg & Si & $\mathbf{C u}$ & $\mathbf{Z n}$ & $\mathbf{F e}$ & Mn \\
\hline & \multicolumn{4}{|c|}{ - } & \multicolumn{4}{|c|}{ - } \\
\hline Controle & 10,30 & 4,60 & 1,50 & 0,63 & 9,00 & 12,27 & 105 & 140 \\
\hline $\mathrm{AC}$ & 10,28 & 4,13 & 1,57 & 0,55 & 8,00 & 16,90 & 88,33 & 79,33 \\
\hline 10 t.ha ${ }^{-1} \mathrm{~PB}$ & $12,63 *$ & 4,90 & 1,53 & 0,71 & 8,00 & 18,00 & 94,00 & 122,00 \\
\hline 20 t.ha ${ }^{-1} \mathrm{~PB}$ & 12,19 & 4,53 & 1,57 & 0,72 & 10,00 & 18,07 & 86,00 & 108,00 \\
\hline 50 t.ha $^{-1} \mathrm{~PB}$ & $15,12 *$ & 4,13 & 1,53 & 0,64 & 11,00 & 18,80 & 120,00 & 104,00 \\
\hline 100 t.ha ${ }^{-1} \mathrm{~PB}$ & $15,67 *$ & 3,53 & 1,53 & $0,78^{*}$ & 11,00 & 19,33 & 120,67 & 100,33 \\
\hline 10 t.ha ${ }^{-1} \mathrm{~PB}+\mathrm{EB}$ & 10,67 & 4,13 & 1,37 & 0,69 & 9,00 & 16,00 & 113,33 & 89,33 \\
\hline 20 t.ha ${ }^{-1} \mathrm{~PB}+\mathrm{EB}$ & 12,20 & 4,63 & 1,53 & 0,64 & 8,67 & 18,93 & 121,33 & 87,67 \\
\hline 50 t.ha $^{-1} \mathrm{~PB}+\mathrm{EB}$ & $12,52 *$ & 4,87 & 1,57 & 0,72 & 11,33 & 18,07 & 165,33 & 74,67 \\
\hline 100 t.ha ${ }^{-1} \mathrm{~PB}+\mathrm{EB}$ & $12,31 *$ & 5,67 & 1,63 & $0,74 *$ & 10,00 & 17,03 & 184,00 & 120,00 \\
\hline Média & 12,13 & 4,42 & 1,52 & 0,66 & 9,69 & 16,61 & 119,24 & 96,83 \\
\hline C.V. $(\%)$ & 8,98 & 21,47 & 7,42 & 11,75 & 19,75 & 12,35 & 27,50 & 25,87 \\
\hline
\end{tabular}

Médias seguidas de * diferem do controle pelo teste de Duncan $(\mathrm{p} \leq 0,05)$.

Os valores observados não apresentaram diferenças entre os tratamentos para o teor de $\mathrm{Cu}, \mathrm{Zn}, \mathrm{Fe} e$ Mn nas folhas do eucalipto (Tabela 3). Apesar de os micronutrientes serem exigidos em pequenas quantidades pelas plantas, deve-se considerar que as práticas culturais tendem a diminuir a disponibilidade desses elementos no solo, como a remoção pelas culturas e a calagem. Assim, a aplicação de pó de rocha pode ser uma maneira de manter os níveis desses nutrientes no solo, pois na sua composição há presença desses elementos (Tabela 1), que podem ser liberados da estrutura dos minerais que compõem a rocha pela 
ação dos fatores do intemperismo (STRAATEN, 2002), podendo ser suficientes para atender a demanda da planta.

\section{CONCLUSÕES}

- Dez meses após a aplicação, o pó de basalto proporcionou aumento no valor do pH para todas as doses estudadas. $\mathrm{O}$ pH do solo, que foi corrigido para 5,5 antes da instalação do experimento, passou a apresentar valor de 6,1 na média dos quatro tratamentos pela aplicação do pó de basalto e de 6,3 na média dos quatro tratamentos com pó de basalto mais esterco bovino. O que pode ser atribuído as quantidades de $\mathrm{CaO}$ e de $\mathrm{MgO}$ presentes no basalto, 8,56 e 4,82\% respectivamente;

- Os teores de Mg e Si trocável no solo foram maiores nos tratamentos com 20, 50 e 100 t.ha $^{-1}$ com pó de basalto mais esterco bovino, que apresentaram em média $1,74 \mathrm{cmol}_{\mathrm{c}} \cdot \mathrm{dm}^{-3}$ para o $\mathrm{Mg} \mathrm{e}$ $13,57 \mathrm{mg} \cdot \mathrm{dm}^{-3}$ para o $\mathrm{Si}$, indicando que o esterco bovino pode ser um material que interfere na dissolução dos minerais e na liberação de $\mathrm{Mg}$ e Si pelo intemperismo biológico. O pó de basalto, em associação ou não com esterco bovino, conferiu teores de $\mathrm{K}$ nas folhas das plantas (média de $\left.12,91 \mathrm{~g} \cdot \mathrm{kg}^{-1}\right)$ maiores que o tratamento-controle $\left(10,30 \mathrm{~g} \cdot \mathrm{kg}^{-1}\right)$;

- Com base nos resultados, conclui-se que a combinação de pó de basalto com esterco bovino pode acelerar a liberação dos minerais da rocha. Doses a partir de 20 t.ha $^{-1}$ de pó de basalto associado ao esterco bovino promoveram aumento no $\mathrm{pH}$ do solo e podem ser fonte de $\mathrm{Mg}$, Si para o solo e K para as plantas de eucalipto, podendo ser utilizado em pequenos viveiros florestais. No entanto, para ser utilizado em larga escala, devem ser realizadas outras avaliações por períodos de tempo mais longos, relacionadas com o desenvolvimento da cultura.

\section{REFERÊNCIAS}

ASSOCIAÇÃO BRASILEIRA DE PRODUTORES DE FLORESTAS PLANTADAS (ABRAF). Anuário estatístico da ABRAF 2010 ano-base 2009/ABRAF. - Brasília, 2010. Disponível em $<$ http://www.abraflor.org.br/estatisticas.asp $>$. Acesso em: 15/08/2010.

ASSOCIAÇÃO NACIONAL PARA A DIFUSÃO DE ADUBOS (ANDA). Disponível em < http://www.anda.org.br/home.aspx $>$. Acesso em 15/08/2009.

BOLLAND, M. D. A.; BAKER, M. J. Powdered granite is not an effective fertilizer for clover and wheat in sandy soils from Western Australia. Nutrient Cycling in Agroecosystems, Dordrecht, v. 5, p. 59 - 68, 2000.

COMISSÃO DE QUÍMICA E FERTILIDADE DO SOLO (CQFSRS/SC). Manual de adubação e de calagem para os Estados do Rio Grande do Sul e Santa Catarina. Porto Alegre: Sociedade Brasileira de Ciência do Solo/Núcleo Regional Sul, 2004. v. 2. 400 p.

ELLIOTT, C. L.; SNYDER, G. H. Autoclave-induced digestion for the colorimetric determination of silicon in rice straw. Journal Agriculture Food Chemical, California, v. 39, p. 1118 - 1119, 1991.

EMPRESA BRASILEIRA DE PESQUISA AGROPECUÁRIA (EMBRAPA). Sistema brasileiro de classificação de solos. Rio de Janeiro: Centro Nacional de Pesquisa de Solos, 2006. v. 2. 306 p.

ESCOSTEGUY, P. A. V.; KLANT, E. Basalto moído como fonte de nutriente. Revista Brasileira de Ciência do Solo, Viçosa, v. 22, p. 11 - 20, 1998.

FERREIRA, E. R. N. C.; ALMEIDA, J. A.; MAFRA, A. L. Pó de basalto, desenvolvimento e nutrição do feijão comum (Phaseolus vulgaris) e propriedades químicas de um Cambissolo Húmico. Revista de Ciências Agroveterinárias, Lages, v. 8, p. 111 - 121, 2009.

FRAGSTEIN, P.; PERTL, W.; VOGTMANN, H. Verwitterungsverhalten silikatischer Gesteinsmehle unter Laborbedingungen. Zeitschrift für Pflanzenernährung und Bodenkunde, Berlin, v.151, p. 141 $146,1988$.

GILLMAN, G. P. The effect of crushed basalt scoria on the cation exchange properties of a highly weathered soil. Soil Science Society of America Journal, Madison, v. 44, p. 465 - 468, 1980. 
GILLMAN, G. P.; BUEKKETT, D. C.; COVENTRY, R. J. A laboratory study of application of basalt dust to highly weathered soils: effects ion soil cation chemistry. Australian Journal of Soil Research, Collingwood, v. 39, p. 799 - 811, 2001.

GONÇALVES, J. L. M. Recomendações de adubação para Eucalyptus, Pinus e espécies típicas da Mata Atlântica. Documentos Florestais, Piracicaba, v. 15, p. 1 - 23, 1995.

GRAÇA, M. E. C.; SHIMIZU, J. Y.; TAVARES, F. R. Capacidade de rebrota e de enraizamento de Eucalyptus benthamii. Boletim de Pesquisa Florestal, Colombo, v. 39, p. 135 - 138, 1999.

HARLEY, A. D.; GILKES, R. J. Factors influencing the release of plant nutrient elements from silicate rock powders: a geochemical overview. Nutrient Cycling in Agroecosystems, Dordrecht, v. 56, p. 11 - 36, 2000.

HIGA, R. C. V. Aspectos ecológicos e silviculturais do Eucalyptus benthamii Maiden et Cambage. Boletim de Pesquisa Florestal, Colombo, v. 38, p. 121 - 123, 1999.

HIGA, R. C. V.; PEREIRA, J. C. D. Usos potenciais do Eucalyptus benthamii Maiden et Cambage. Colombo: Embrapa Florestas, 2003. v. 100. 5 p.

KIEHL, E. J. Fertilizantes orgânicos. São Paulo: Agronômica Ceres, 1985. v. 1. 492 p.

KNAPIK, J. G.; ANGELO, A. C. Pó de basalto e esterco equino na produção de Mudas de Prunus sellowii Koehne (Rosaceae). Floresta, Curitiba, v. 37, p. 427 - 436, 2007.

LEONARDOS, O. H.; FYLE, W. S.; KROMBERG, B. Rochagem: método de aumento de fertilidade em solos lixiviados e arenosos. In: CONGRESSO BRASILEIRO DE GEOLOGIA, 29., 1976, Ouro Preto. Anais do... São Paulo, 1976. p. 137 - 145.

MARSCHNER, H. Root-induced changes in the availability of micronutrients in the rhizosphere. In: WAISEL, Y.; ESHEL, A.; KAFKAFI, V. Plants roots: The hidden half. New York, 1991. p. 503 - 528.

MARSCHNER, H. Mineral nutrition in higher plants. San Diego: Academic Press, 1995. v. 1. 889 p.

OSTERROHT, M. V. Rochagem para quê? Agroecologia Hoje, Botucatu, v. 20, p. 12 - 15, 2003.

PERRENOUD, S. Potassium and plant health. 2 ed. Berna: International Potash Institute, 1990. 363 p.

QAFOKU, N. P.; SUMNER, M. E.; RADCLIFFE, D. E. Anion transport in columns of variable charge subsoils: nitrate and chloride. Journal Environmental Quality, Madison, v. 29, p. 484 - 493, 2000.

QUAGGIO, J. A. Acidez e calagem em solos tropicais. Campinas: Instituto Agronômico de Campinas, 2000. v. 1, $111 \mathrm{p}$.

RESENDE, M.; CURI, N.; REZENDE, S. B.; CORRÊA, G. F. Pedologia: base para distinção de ambientes. Viçosa: Neput, 2002. 338 p.

SILVEIRA, R. L. V. A.; HIGASHI, E. N.; GONÇALVES, A. N.; MOREIRA, A. Avaliação do estado nutricional do Eucalyptus: diagnose visual, foliar e suas interpretações. In: GONÇALVES, J. L. M.; BENEDETTI, V. Nutrição e fertilização florestal. Piracicaba, 2000. p. 79 - 104.

STEVEnSON, F. J.; COLE, M. A. Cycles of soil: carbon, nitrogen, phosphorus, sulfur and micronutrients. New York: John Wiley, 1999, v. 1.427 p.

STRAATEN, P. Rocks for crops: agrominerals of Sub-Saharan Africa. Nairobi: ICRAF, 2002. v. 1. 338 p.

TEDESCO, M. J.; GIANELLO, C.; BISSANI, C. A.; BOHNEN, H.; VOLKWEISS, S. J. Análises de solos, plantas e outros materiais. Porto Alegre: Universidade Federal do Rio Grande do Sul, 1995. v. 1. 171 p.

THEODORO, S. H.; LEONARDOS, O. H. The use of rocks to improve family agriculture in Brazil. Anais da Academia Brasileira de Ciências, Rio de Janeiro, v. 78, p. 721 - 730, 2006.

WILPERT, K.; LUKES, M. Ecochemical effects of phonolite rock powder, dolomite and potassium sulphate in a spruce stand on an acidified glacial loam. Nutrient Cycling in Agroecosystems, Dordrecht, v. 65 , p. $115-127,2003$. 